\title{
Interpretation of sweep frequency response analysis traces on inter-turn short circuit fault
}

\author{
N. F. M. Yasid ${ }^{1}$, A. A. Alawady ${ }^{2}$, M. F. M. Yousof ${ }^{3}$, S. Al-Ameri ${ }^{4}$, M. S. Kamarudin ${ }^{5}$ \\ 1,2,3,4,5 Faculty of Electrical and Electronic Engineering, Universiti Tun Hussein Onn Malaysia, Malaysia \\ ${ }^{2}$ Department of Computer Technical Engineering, Islamic University, Iraq
}

\begin{tabular}{l} 
Article Info \\
\hline Article history: \\
Received Jun 27, 20 \\
Revised Nov 12, 2019 \\
Accepted Dec 3, 2019 \\
\hline Keywords: \\
Frequency response \\
Short circuit fault \\
Transformer \\
Winding
\end{tabular}

\begin{abstract}
Sweep frequency response analysis (SFRA) is a reliable method for detection and diagnosis of faults in the active part of transformers. However, although SFRA is widely employed, the interpretation of SFRA signature is still a challenge and require experts to analyse them. This is due to lack of guideline and standard for SFRA signature interpretation and clarification. This paper presents the interpretation of SFRA signature by classification and quantification on inter-turn short circuit fault on the transformer winding. The short-circuited turns fault on HV winding phase "A" was practically simulated on three different units of three-phase transformers. The results of simulated fault are presented and discussed. A conclusion was drawn which provides the interpretation of the SFRA response due to inter-turn short circuit fault case by using a statistical indicator which is NCEPRI algorithm.
\end{abstract}

This is an open access article under the CC BY-SA license.

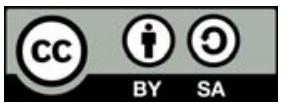

\section{Corresponding Author:}

Nurul Farahwahida Md Yasid,

Department of Electrical and Electronic Engineering,

Universiti Tun Hussein Onn Malaysia (UTHM),

86400, Parit Raja, Batu Pahat, Johor, Malaysia.

Email: farahwahida.yasid@gmail.com

\section{INTRODUCTION}

SFRA is a non-intrusive electrical test which avoid opening a transformer tank to assess their electrical and mechanical condition. Opening the tank will cause oil degasification and dehydration, which can minimize the impact on system operation and loss of supply to customers. Therefore, using SFRA can save millions of ringgits in timely maintenance. Besides, it can deliver valuable information regarding the mechanical as well as the electrical conditions of the transformer [1]. SFRA test can be the key in making decision to scrap or reenergize a transformer. To obtain information from SFRA test, it is necessary to make sure the measurements are correct and accurate interpretation is given.

Comprehensive study of previous literature along with experience performing numerous SFRA test can provide an understanding to interpret the SFRA results. Since SFRA interpretation is based on experience, such records of previous measurements are great importance when interpreting SFRA responses. This paper is presenting the frequency response interpretation of faulty transformer winding due to inter-turn short circuit (SC) fault. The measurements in this study were conducted on three units of three-phase transformers at a local research utility company. The end-to-end open circuit test is performed in this study. Three artificial inter-turn SC faults were created by shorting the windings. The findings in this study are then compared with findings from other previous literatures. 


\section{BIBLIOGRAPHY SURVEY}

The occurrence of SC fault is quite rare. However, the probability is increased during the transformer lifetime, typically when reaching 50 years [2]. In spite of this, according to [3] about $40 \%$ of the transformer faults initiated by huge impact of short circuit annually. Reference [4] also mentioned that fault at winding usually initiated by short circuited. One of the sources which contributes to SC fault is the breakdown of insulation between turns in the winding. Insulation breakdown usually occurs due to high voltage which is above the rated value. The huge current excites an extensive leakage magnetic field and generate a huge dynamical electromagnetic force on the windings. At certain point, the physical strength reaches the point of breaking the winding insulation and unable to tolerate the forces. The breakdown of the insulations could cause flashover and eventually causes the inter-winding short circuit faults. Besides that, according to [5], since the windings are made of copper, thermal losses occur. These thermal losses make hotspots in the winding. This over time reduces the physical strength and eventually initiate the breaking of insulation in the winding. Ultimately this causes short circuit fault on the winding. Another factors to consider which cause this fault are irregular repair, improper or lack of maintenance, deterioration, and manufacturing defect within the transformers. An early detection of low level SC faults is crucial, because if not quickly detected, they usually develop into more serious damage to the transformers. Short circuit is a serious fault which have initiate various transformer breakdowns especially to three-phase transformers [6].

There are three basic categories of SC faults on transformer winding as mentioned in [7]. These are, winding-to-ground, winding-to-winding, and turn-to-turn faults. Turn-to-turn or inter-turn SC fault is found to be mostly occurring. Figure 1 shows the inter-turn SC fault in a transformer winding.

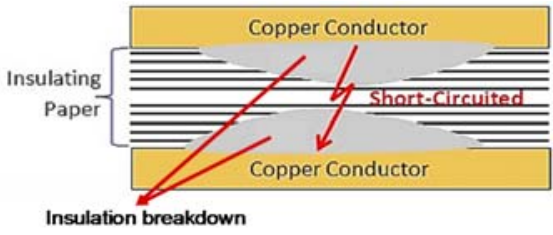

(a)

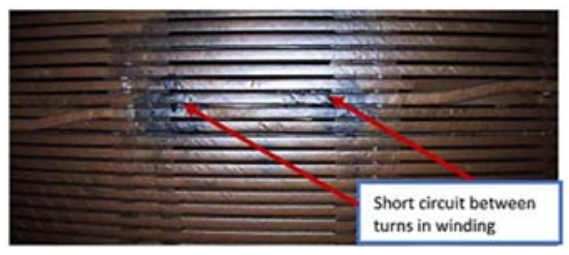

(b)

Figure 1. Inter-turn short circuit fault for (a) Insulation breakdown (b) An actual case [8]

Basically, SC in winding causes the electrical length of the winding to be reduced. Therefore, the winding natural frequencies shall either changed or remain almost unchanged if the SC fault occurs. This is an important property that can be used as the main sign of the winding SC fault. In the case of core-typed three-phase transformers, the internal SC fault will cause an extensive increase of first antiresonance in the low frequency (LF) response [9]. The first antiresonance frequency is typically from hundreds of $\mathrm{Hz}$ up to few $\mathrm{kHz}$. However, it may increase significantly depending on the severity of the SC faults as shown in Figure 2(a). Nevertheless, the increase of the frequency is not totally an indication of a SC occurred just in the winding under measurement. This is because the scenario give effect to all first antiresonance responses of windings on the same core [10]. Reference [10] presented by S. Larin shows that short circuiting the LV2 give effect to the measured response of LV1 as illustrated in Figure 2(b). This is due to the mutual coupling between the HV and LV winding when transformer is energized. Table 1 presents the bibliography survey showing the effect of internal SC fault in the frequency response.

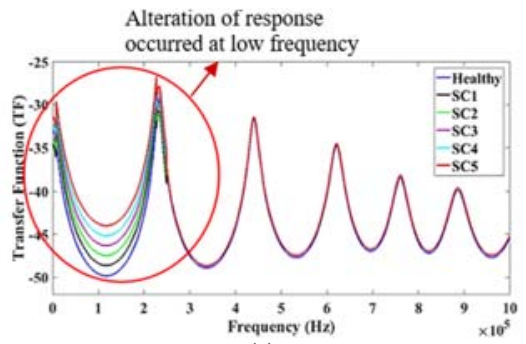

(a)

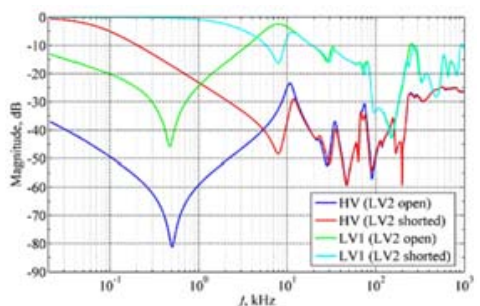

(b)

Figure 2. Impact of SC fault on the SFRA signature presented in (a) [9], (b) [10]

Int J Pow Elec \& Dri Syst Vol. 11, No. 1, Mar 2020 : $309-316$ 
Table 1. Dominant frequency range of SFRA due to short circuit fault from bibliography survey

\begin{tabular}{|c|c|}
\hline References & Dominant frequency range $(\mathrm{Hz})$ \\
\hline [11] Devadiga et al. 2018 & $\begin{array}{l}\text { Low and medium frequency (Significant reduction in } \\
\text { impedance and a shift in resonance peaks towards high } \\
\text { frequencies) }\end{array}$ \\
\hline [12] M.Gutten, Richard Janura, Milan Sebok, Daniel Korenciak, 2016 & Low to medium frequencies (up to $100 \mathrm{kHz}$ ) \\
\hline [13] Aljohani \& Abu-Siada, 2015 & $\begin{array}{l}\text { High levels of SC have a visible impact along the entire } \\
\text { frequency range and influence the resonance frequencies } \\
\text { in high frequency range. }\end{array}$ \\
\hline [14] Pandya \& Parekh, 2014 & Low frequency range $(1 \mathrm{kHz}-10 \mathrm{kHz})$ \\
\hline [15] Bagheri, Naderi, Blackburn, Phung, \& Liu, 2012 & Low frequency region $(20 \mathrm{~Hz}-40 \mathrm{kHz})$ \\
\hline [16] Ilampoornan, Vikash, \& Newton, 2012 & Low frequency region, \\
\hline [17] Firoozi, Kharezi, Rahimpour, \& Shams, 2011 & low frequency range (between $50 \mathrm{~Hz}$ and $2 \mathrm{kHz}$ ) \\
\hline [18] Behjat, Vahedi, Setayeshmehr, Borsi, \& Gockenbach, 2011 & low-frequency deviation (frequency range below $1 \mathrm{kHz}$ ) \\
\hline [19] Contin, Rabach, Borghetto, \& Nigris, 2011 & Low frequency range (up to $10 \mathrm{kHz}$ ) \\
\hline [20] Bigdeli, Vakilian, \& Rahimpour, 2011 & low frequency range of $(10-100 \mathrm{kHz})$ \\
\hline [21] Picher 2008 & Low frequency range \\
\hline
\end{tabular}

Table 1 clearly shows the main effect of internal SC is the shift of frequency response in the low frequency (LF) region. According to [18], the key point to understand the LF deviation caused by shorted turns can be found in the explanation of Faraday's law in shorted turns. The law states that the electromotive force (emf) induced in a turn is equal to the rate of variation of the electromagnetic flux inside it [17].

\section{TRANSFORMER USED IN EXPERIMENTAL WORK}

Three units of three-phase, core-typed transformers were used in this study. The first experimental transformer is an open terminal transformer that is connected as Dyn 11 winding configuration. The second and third experimental transformers are Dyn11, 500kVA and 100kVA distribution transformer. These two tested units are oil-typed transformer which were taken out of service. In this paper, the focus is on the DeltaWye connection which is most frequently used for distribution transformers. According to [5], winding SC usually occurs to distribution transformers. This is because, the current flow in the primary side winding of the transformer undergoes electromagnetic induction voltage which is stepped down. The current is then stepped up in the secondary winding. Due to this, the windings have to tolerate dielectric, thermal and also mechanical stress. This is one of the factors that contribute winding short circuit fault in the LV winding of the transformer. Table 2, Table 3 and Table 4 show the basic details of the transformers used in this study.

Table 2. Details of 360VA transformer

\begin{tabular}{ll}
\hline \multicolumn{1}{c}{ Attributes } & \multicolumn{1}{c}{ Details } \\
\hline Model & KTS 10MT-3/360 VA \\
Capacity & Three-phase, \\
experimental transformer of 360VA \\
High voltage winding & 240 V Delta connected \\
Low voltage winding & $3 \times 60$ V Star connected \\
Current & $2 \mathrm{~A}$ \\
\hline
\end{tabular}

Table 3. Details of 500kVA transformer

\begin{tabular}{ll}
\hline \multicolumn{1}{c}{ Attributes } & \multicolumn{1}{c}{ Details } \\
\hline Model & ONAN TRANSFORMER \\
Serial No. & DRG. No. MTM 11/3/1-D \\
Manufactured date & T/84/5168 \\
Capacity & Three-phase, $50 \mathrm{~Hz}, 500 \mathrm{kVA}$ \\
Total mass & $2668 \mathrm{Kg}$ \\
\hline
\end{tabular}

Table 4. Details of 100kVA transformer

\begin{tabular}{cc}
\hline Attributes & Details \\
\hline Model & MTM TRANSFORMER \\
Serial No. & $1 / 44 / 1160$ \\
Manufactured date & $05 / 09 / 1994$ \\
Capacity & Three-phase, $50 \mathrm{~Hz}, 100 \mathrm{kVA}$ \\
Total mass & $620 \mathrm{Kg}$ \\
\hline
\end{tabular}

\section{TEST METHODOLOGY}

Three transformers were used in this study by creating artificial SC fault in the windings. The purpose is to study the variation that might occur in the transformer frequency response due to this fault. In order to diagnose short circuit fault, SFRA measurement is performed before and after the fault has occurred. The comparison of responses before and after fault is then observed and analyzed. This is conducted per phase using end-to-end open circuit configuration. As the term allude to, per phase measurement aimed for a particular phase at one time. In this paper, SFRA measurement is performed on phase A only which is the same phase as the SC fault is applied. Figure 4 shows SC fault which was created on the first transformer.

Interpretation of sweep frequency response analysis traces on inter-turn short circuit fault (N. F. M. Yasid) 


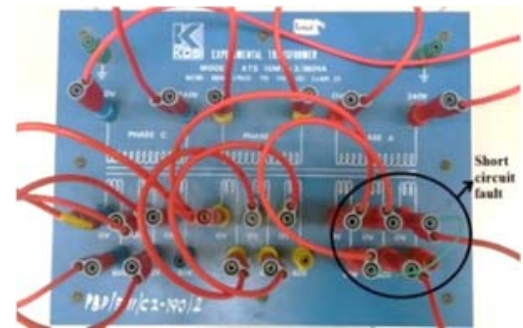

(a)

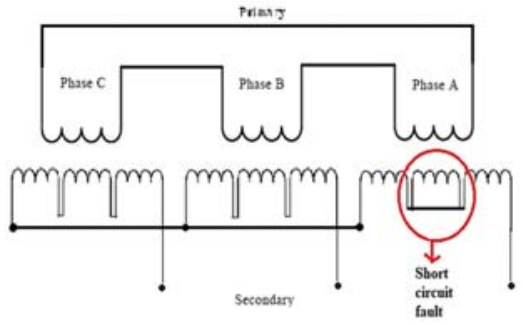

(b)

Figure 3. (a) SC fault simulated on phase A of LV winding, (b) Schematic diagram of SC fault

The open terminal transformer has two windings (HV and LV) per phase. The LV winding itself has three separated and identical windings. Since these windings are not connected, SC fault is created by shorting the middle section of the LV winding. Figure 3(a) shows a short and thin cable connecting the LV windings. This causes $1 / 3$ of the total LV winding to be shorted. Figure 3 (b) shows the schematic diagram of the SC fault on phase A of LV winding.

The second short circuit was simulated on the 500kVA distribution transformer as shown in Figure 4(a) and the last short circuit fault is simulated on 100kVA transformer as shown in Figure 4(b).

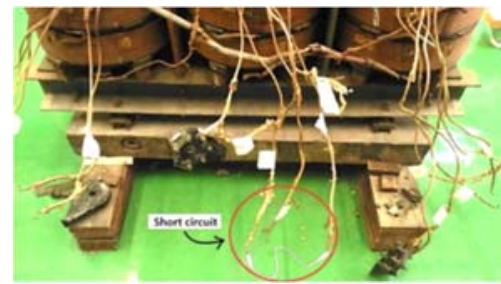

(a)

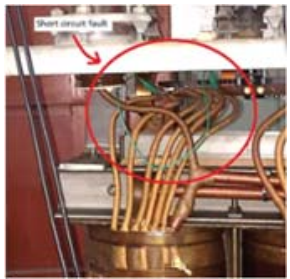

(b)

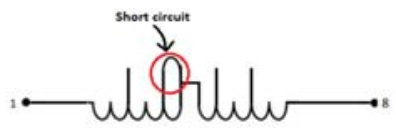

(c)

Figure 4. SC fault simulated on transformer tap 3-4 phase A (a) 500kVA transformer, (b) 100kVA transformer, (c) Schematic diagram of the SC fault

As shown in Figure 4(a), the SC fault was created by connecting a wire cable to the exposed transformer tap in the HV winding. The exposed tap is labelled according to their tap references. This causes short fault and the total turns of the HV winding of that phase to reduce. On the other hand, Figure 4(b) shows the SC fault is created by connecting a cable to the transformer taps in the HV winding of $100 \mathrm{kVA}$ transformer. Figure 4(c) shows the schematic diagram of winding with short circuit at tap 3-4 for both transformers.

\section{SFRA RESPONSE DUE TO SC FAULT}

The results of the three artificial SC faults are presented in this section. The SFRA measurements on $\mathrm{HV}$ windings of three tested transformers are shown in Figure 5, Figure 6 and Figure 7. Each of the figure shows two traces measured during normal and faulty operating conditions of the transformers. The response is divided into three frequency regions. As referred to [22], the frequency regions are not fix at a certain frequency but should be defined according to the shape of the response. The frequency response will be different depending on the structure, configuration, winding type and power rating of the transformer.

From the measured responses of all three transformers (Figure 5, Figure 6 and Figure 7), the main effect of SC fault is the shift of response at the low frequency (LF) region towards higher frequencies. The 
shifting is more obvious at LF region and early part of medium frequency (MF) region. Clearly, the faulty traces for the tested transformers show a similar shifting or deviation.

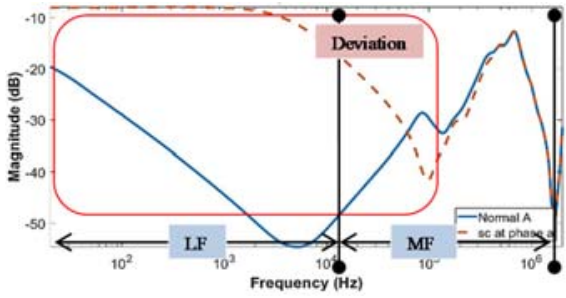

Figure 5. FRA response of normal and short circuit winding of 360VA transformer

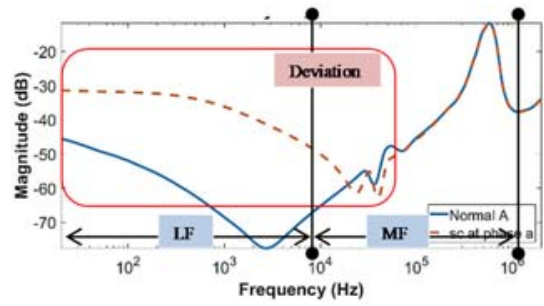

Figure 6. FRA response of normal and short circuit winding of 500kVA transformer

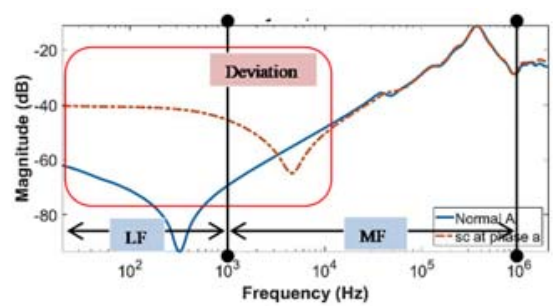

Figure 7. FRA response of normal and short circuit winding of 100kVA transformer

It is clear from the transformers responses, the magnitude $(\mathrm{dB})$ increases and the first resonances at LF region shifted towards higher frequencies. The key point to understand the LF deviations can be found in the explanation of change in the magnetizing characteristics of the transformer initiated by shorted turns. Basically, the impedance of winding is small, meanwhile the impedance of the core is extremely high. This parameter governs the LF region [22]. Therefore, the input signal is dominated by the core. However, as the SC is added, changes in the core are detected. It causes the effect of core saturation to reduce and significantly decreased the magnetizing inductance. Due to this reason, the response of the LF region rises or shifted towards higher frequencies, and it has higher starting $\mathrm{dB}$ value than response of healthy winding. This condition can also be explained by using (1).

$\Phi \propto \mathrm{NI}$

The (1) represents the amount of flux, $\Phi$ which is directly proportional to the product of the number of turns, $\mathrm{N}$ and the current flow in the winding, I. In general, the internal short circuit leads to a reduction of the electrical length of the winding, meaning reduction in the number of turns [10]. This is an important property that can be used as one of the main signs of the internal short-circuit fault in the winding. As the number of turns reduces, the flux produced in the coil also reduced significantly. Therefore, during this fault, the magnetizing inductance of the right-side limb (Phase A) is drastically reduced and caused the magnitude of the response in LF region to increase. The large amount of shift is affected by the reduction of magnetizing inductance in the core limb. Which is caused by the SC fault in the secondary winding of the same phase as the SFRA measurement conducted.

\section{FAILURE INTERPRETATION USING NCEPRI ALGORITHM}

In order to analyse and interpret the frequency response, a mathematical indicator is applied. Usually, mathematical or statistical indicator is a method to measure the similarities between two frequency responses. This is performed rather than by visually comparing from the figures only. This method can provide a single value on the extent of the variation between them. To achieve more accuracy in diagnosing the requency response, many statistical and mathematical indices have been proposed in the literature for SFRA application. For example, correlation coefficient (CC), absolute sum of logarithmic error (ASLE), min-max (MM) ratio and root mean square error (RMSE). In this study, an algorithm proposed in [23] which

Interpretation of sweep frequency response analysis traces on inter-turn short circuit fault (N. F. M. Yasid) 
is North China Electric Power Research Institute (NCEPRI) algorithm has been used to analyse the responses. This algorithm is proposed by a group of researcher in [24] as a mathematical indices to analyse the frequency responses.

\subsection{NCEPRI algorithm}

The NCEPRI algorithm estimates the correspondence of frequency responses by calculating the effective deviation (ED) of two transfer functions using (2).

$$
E_{12}=\frac{1}{N} \sum_{i=1}^{N}\left(T F_{1 i}-T F_{2 i}\right)^{2}
$$

Where $T F_{1 i}$ and $T F_{2 i}$ are the reference and compared transfer functions or frequency responses. $N$ is the number of data. Table 5 shows the interpretation criteria for NCEPRI algorithm. According to NCEPRI standard proposed in [23], the frequency ranges required for the winding assessment factor, $\mathrm{E}_{12}$ can be devided into three as shown in Table 6. They are the high-voltage (HV), low-voltage (LV) and tertiary voltage (TV).

Table 5. Failure interpretation criteria of NCEPRI

\begin{tabular}{cc}
\multicolumn{2}{c}{ algorithm } \\
\hline Distortion level & Factor $(\mathrm{dB})$ \\
\hline Normal condition & $\mathrm{E}_{12}<3.5$ \\
Slight distortion & $3.5>\mathrm{E}_{12} \geq 7.0$ \\
Serious distortion & $\mathrm{E}_{12}>7.0$ \\
\hline
\end{tabular}

Table 6. Frequency range defined in [22]

\begin{tabular}{cc}
\hline Winding & Frequencies \\
\hline HV & $10 \mathrm{kHz}-515 \mathrm{kHz}$ \\
$\mathrm{TV}$ & $10 \mathrm{kHz}-600 \mathrm{kHz}$ \\
\hline
\end{tabular}

However, [22] has found that using large frequency ranges for the analysis reduces the sensitivity and makes the failure classification difficult. The frequency regions do not fix at a certain frequency only but should be defined according to the shape of the frequency response. During evaluating the frequency response, user should modify and adjust the frequency region according to the response shape. It can also be generally associated with a much narrower frequency range to achieve higher sensitivity.

\subsection{Response analysis using NCEPRI algorithm}

Visually comparing all three measurements, Figure 5, Figure 6 and Figure 7 clearly reveal some important features. The inter-turn SC fault cause obvious variations in low and beginning of the medium frequency region. NCEPRI algorithm is applied to measure the similarity between the normal and faulty winding responses. Table 7 shows the frequency ranges defined according to the response features. On the other hand, Table 8 reveals the fault assessment factor of the winding deformation in term of effective deviation between the responses.

Table 7. Three frequency ranges according to size

\begin{tabular}{cccc}
\hline \multirow{2}{*}{ Transformer } & LF & Frequency range \\
& MF & HF \\
\hline $360 \mathrm{VA}$ & $20 \mathrm{~Hz}-1.5 \mathrm{kHz}$ & $1.5 \mathrm{kHz}-1.6 \mathrm{MHz}$ & $1.6 \mathrm{MHz}-2 \mathrm{MHz}$ \\
$500 \mathrm{kVA}$ & $20 \mathrm{~Hz}-9 \mathrm{kHz}$ & $9 \mathrm{kHz}-1.5 \mathrm{MHz}$ & $1.5 \mathrm{MHz}-2 \mathrm{MHz}$ \\
$100 \mathrm{kVA}$ & $20 \mathrm{~Hz}-1 \mathrm{kHz}$ & $1 \mathrm{kHz}-1 \mathrm{MHz}$ & $1 \mathrm{MHz}-2 \mathrm{MHz}$ \\
\hline
\end{tabular}

Table 8. Fault assessment according to

\begin{tabular}{cccc}
\multicolumn{4}{c}{ NCEPRI algorithm } \\
\hline \multirow{4}{*}{ Transformer } & \multicolumn{3}{c}{ Fault assessment Factor, } \\
& EF & MF $\mathrm{dB}$ & $\mathrm{HF}$ \\
\hline 360VA & 33.846 & 9.942 & 0.046 \\
$500 \mathrm{kVA}$ & 27.867 & 5.162 & 0.135 \\
100kVA & 35.598 & 6.653 & 1.173 \\
\hline
\end{tabular}

The frequency ranges are different as it is defined according to the response shapes. Obviously, they will be different because the transformer has a different power rating. The frequency ranges can be referred as in Table 7 and illustrated as in Figure 5, 6 and 7 respectively. From Table 8, it can be seen that the fault assessment factor for the low frequency (LF) region is very high. Clearly, all the values are above beyond 7 . Therefore, the LF region is considered as serious distorted as referred to Table 5. Meanwhile, $\mathrm{E}_{12}$ of MF region for $100 \mathrm{kVA}$ and $500 \mathrm{kVA}$ is considered as slight distortion. This is differently to $360 \mathrm{VA}$ which shows a serious distortion. However, the degree of distortion is not as high as in the LF region. On the other hand, the high frequency $(\mathrm{HF})$ region is considered as in normal condition. 


\section{CONCLUSION}

It was proven that winding short circuit fault gives a significant change on the SFRA response. Findings in this paper conclude that the fault causes serious alteration in the low frequency region and slight alteration in the medium frequency region. The main sign is the significant shift of response in low frequency region towards higher frequencies. The initial magnitude of faulty response is also higher in $\mathrm{dB}$ compared to normal response. Besides that, failure interpretation using the NCEPRI algorithm also has proven the finding. It shows that the low frequency region has the highest value of assessment factor, $\mathrm{E}_{12}$ and indicate serious distortion. The level of distortion reduces as the frequencies increases. This prove that the inter-turn short circuit fault gives a significant effect to low frequency region. Slightly affect the medium frequency region when it is severe and not effecting in the high frequency region.

\section{ACKNOWLEDGEMENTS}

The authors would like to thank the TNB Research, for the provision of test facilities and great support for the experimental tests. This work is supported financially by the Islamic University research grant PRG-IUNAJAF-2019.

\section{REFERENCES}

[1] M. F. M. Yousof, "Frequency response analysis for transformer winding condition monitoring," University of Queensland, 2015.

[2] G. D. Hermann, "Short-circuit withstand capability of power transformers," 2015.

[3] D. Zhou, Z. Li, C. Ke and Z. Hao, "Simulation of transformer windings mechanical characteristics during the external short-circuit fault," IEEE conference on Electric Utility Deregulation and Restructuring and Power Technologies, pp. 1068-1073, 2015.

[4] G. M. Kennedy, A. J. Mcgrail, J. A. Lapworth and D. Engineering, "Transformer sweep frequency response analysis (SFRA)," Energize, pp. 28-33, 2007.

[5] S. T. Jan, R. Afzal and A. Z. Khan, "Transformer failures, causes \& impact," International Conference Data Mining, Civil and Mechanical Engineering (ICDMCME'2015), Vol. 4, No. 4, pp. 49-52, 2015.

[6] H. Zhang, et al., "Dynamic deformation analysis of power transformer windings in short-circuit fault by FEM," IEEE Transactions on Applied Superconductivity, Vol. 24, No. 3, pp. 1-4, 2014.

[7] B. Kasztenny, M. Thompson and N. Fischer, "Fundamentals of short-circuit protection for transformers," 2010 63rd Annual Conference for Protective Relay Engineers, Vol. 2, No. 3, pp. 4-7, 2010.

[8] R. Murugan and R. Ramasamy, "Understanding the power transformer component failures for health index-based maintenance planning in electric utilities," Engineering Failure Analysis, Vol. 96, No. July 2018, pp. 274-288, 2019.

[9] A. R. Abbasi, M. R. Mahmoudi and Z. Avazzadeh, "Diagnosis and clustering of power transformer winding fault types by cross-correlation and clustering analysis of FRA results," IET Generation, Transmission \& Distribution, vol. 12(19), pp. 4301-4309, 2018.

[10] V. S. Larin, "Internal short-circuits faults localization in transformer windings using FRA and natural frequencies deviation patterns," CIGRE Study Committee A2 COLLOQUIUM, No. October, pp. 1-14, 2017.

[11] A. A. Devadiga, N. Harid, H. Griffiths, N. Al Sayari, B. Barkat and S. Jayaram, "Winding turn-to-turn short-circuit diagnosis using FRA method: Sensitivity of measurement configuration," IET Science, Measurement \& Technology, 2018.

[12] M. K. M. Gutten, Richard Janura, Milan Sebok, Daniel Korenciak, "Measurement of short circuit effects on transformer winding with SFRA method and impact test," Metrology and Measurement System, Vol. 23, No. 4, pp. 521-529, 2016

[13] O. Aljohani and A. Abu-Siada, "Minimum detection of power transformer short circuit fault using frequency response analysis," IEEE, 2015.

[14] A. A. Pandya and B. R. Parekh, "Interpretation of sweep frequency response analysis (SFRA) traces for the open circuit and short circuit winding fault damages of the power transformer," International Journal of Electrical Power and Energy Systems, Vol. 62, pp. 890-896, 2014.

[15] M. Bagheri, M. S. Naderi, T. Blackburn, B. T. Phung and Z. Liu, "Frequency response analysis to recognize inductance variation in transformer due to internal short circuit," 10th International Power and Energy Conference, IPEC 2012, pp. 677-681, 2012.

[16] M. K. Ilampoornan, M. Vikash and F. B. I. L. Newton, "Transformer fault detection by frequency response analysis," IOSR Journal of Electrical and Electronic Engineering (IOSRJEEE), vol. 1, No. 4, pp. 27-32, 2012.

[17] H. Firoozi, M. Kharezi, H. Rahimpour and M. Shams, "Transformer fault diagnosis using frequency response analysis - practical studies," Power and Energy Engineering Conference (APPEEC), 2011 Asia-Pacific, pp. 1-4, 2011.

[18] V. Behjat, A. Vahedi, A. Setayeshmehr, H. Borsi and E. Gockenbach, "Diagnosing shorted turns on the windings of power transformers based upon online FRA using capacitive and inductive couplings," IEEE Transactions on Power Delivery, Vol. 26, No. 4, pp. 2123-2133, 2011. 
[19] A. Contin, G. Rabach, J. Borghetto and M. De Nigris, "Frequency-response analysis of power transformers by means of fuzzy tools," IEEE Transactions on Dielectrics and Electrical Insulation, Vol. 18, No. 3, pp. 900-909, 2011.

[20] M. Bigdeli, M. Vakilian and E. Rahimpour, "A new method for detection and evaluation of winding mechanical faults in transformer through transfer function measurements," Advances in Electrical and Computer Engineering, vol. 11, No. 2, pp. 23-30, 2011.

[21] P. Picher, "Mechanical condition assessment of transformer windings using frequency response analysis (FRA)," Cigre, no. April. pp. 30-34, 2008.

[22] J. C. Gonzales and E. E. Mombello, "Automatic detection of frequency ranges of power transformer transfer functions for evaluation by mathematical indicators," Transmission and Distribution: Latin America Conference and Exposition (T\&D-LA), IEEE, vol. 978, No. 1, pp. 1-8, 2012.

[23] OMICRON, "FRAnalyzer user manual - SFRA analyzer for power transformer winding diagnosis," 2006.

[24] NCEPRI, "Application guideline for transformer winding distortion test technology," 1999.

\section{BIOGRAPHIES OF AUTHORS}
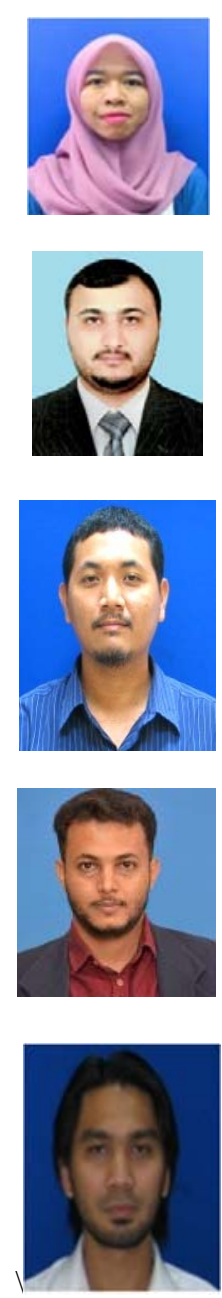

Nurul Farahwahida bt Md Yasid received her bachelor's degree in Electrical Engineering from Universiti Tun Hussein Onn Malaysia (UTHM) in 2017. She is currently pursuing her Master degree in Electrical Engineering at Universiti Tun Hussein Onn Malaysia. Her research interest include high voltage equipment and condition monitoring of transformer using FRA.

Ahmed Allawy Mohammed Alawady received B.Eng. in Electrical from University of Kufa, Iraq in 2007. He received M.Eng. in Electrical power system from (SHUATS), India in 2012. He is a lecturer at the Department of Computer Technical Engineering, Collage of Technical Engineering at Islamic University, Iraq since 2007. Currently he is pursuing Ph.D. Degree in Universiti Tun Hussein Onn Malaysia. His research is on motors faults detection using FRA.

Dr. Mohd Fairouz Mohd Yousof obtained Dip. Eng, B. Eng and M. Eng from Universiti Teknologi Malaysia. He completed his Ph.D. study in 2015 from The University of Queensland, Australia and currently is a lecturer at the Department of Electrical Power Engineering, Universiti Tun Hussein Onn Malaysia (UTHM). He was a visiting researcher for a year with TNB Research in 2018. His main research is condition-based monitoring and assessment of power transformer and rotating machine. He is actively involved with projects from local and international industries.

Salem Mgammal Awadh Nasser Al-Ameri received B.Eng in Mechatronics Engineering from Asia Pacific University (APU) in 2012. He received M.Eng in Electrical Power from Universiti Tun Hussein Onn Malaysia (UTHM) from 2014 to 2016. Currently he is pursuing Ph.D. Degree in Universiti Tun Hussein Onn Malaysia. His research is on transformer monitoring using FRA.

Associate Professor Ir. Dr. Muhammad Saufi Kamarudin obtained his B.Eng. and M.Eng. Degrees in Electrical Engineering (Power) from Universiti Teknologi Malaysia (UTM) in 2003 and 2005, respectively, and a Ph.D. in High Voltage Engineering from Cardiff University, UK, in 2014. Currently, he is an associate professor in the Faculty of Electrical \& Electronic Engineering at Universiti Tun Hussein Onn Malaysia (UTHM). His research interests include gas discharges, high voltage surge arresters, dielectrics and electrical insulation system. He is registered with the Board of Engineers Malaysia (BEM). 